\title{
Supernova remnants in the central starburst region of M82
}

\author{
D. Fenech* T.W.B. Muxlow, R.J. Beswick, A. Pedlar, M.K. Argo, W.M. Trotman \\ Jodrell Bank Observatory \\ E-mail: dfenech@jb.man.ac.uk
}

\begin{abstract}
Global VLBI observations of the central kiloparsec of M82 at $1.6 \mathrm{GHz}$, attaining an angular resolution of 4 mas, were made on 3 Mar 2005. We present detailed images of four of the brightest, most compact supernova remnants as seen in these observations. These data are the fifth epoch of 1.6-GHz VLBI observations of M82, the first of which was made in December 1986, enabling study of these compact sources over a 19 year timescale. We will also discuss MERLIN 1.6-GHz observations made simultaneously, which have been combined with the global VLBI data, to produce high fidelity images of a large number of the known supernova remnants and HII regions in M82.
\end{abstract}

8th European VLBI Network Symposium

September 26-29, 2006

Toruń, Poland

\footnotetext{
*Speaker.
} 


\section{Introduction}

M82 is considered to be the archetypal starburst galaxy and at a distance of $3.2 \mathrm{Mpc}$ is one of the closest [1]. As a consequence, it makes an ideal study ground of an extragalactic starburst environment. The first radio observations of this galaxy were made in the 1960's [2] and subsequent high resolution observations in 1972 [3] revealed a population of compact sources within the central kiloparsec. These sources were initially proposed to be either radio supernovae or supernova remnants (SNR) [4 5], but the subsequent lack of radio variability distinguished them as SNR. Radio interferometry techniques are ideal for observing the SNR within this galaxy for several reasons. The extinction due to dust is extremely high, making it very difficult to observe these sources at optical wavelengths. All of the sources are observed with the same angular resolution and sensitivity, and the relative distance to the individual SNR will only differ by $\sim 0.1 \%$ due to the large distance to the galaxy in question. Therefore, this population of sources has been observed using several of the world's interferometry networks including the VLA, MERLIN and global VLBI, and there are now known to be over 50 discrete sources present in the central kiloparsec, all resolved by either MERLIN [6, 7, 8, 9] and/or VLBI [10, 11, 12]. They form a population of sources brighter, and more compact than Cassiopeia A and so are considered to be young and rapidly evolving SNR.

\section{Global VLBI 1.6-GHz observations}

VLBI observations of the five most compact sources in M82 provide the opportunity to monitor SNR in their environment in great detail. In December 1986, the first of now five epochs of observations were carried out using four of the EVN antennas. Subsequent observations were made using the EVN in June 1997 [10] and using global VLBI in November 1998 and February 2001 [11, 12]. These five epochs of data have been used to image and monitor the expansion of the source $43.31+59.2$, which is a young rapidly evolving supernova remnant with an expected birth date $\sim 1960$, (see section 2.2). The global VLBI observations have also been used to observe the most compact source $41.95+57.5$. The 15 mas beam of the EVN observations does not fully resolve this source and, as a result, only the global VLBI observations have been used to monitor its evolution on scales of a few mas, corresponding to $\sim 0.05 \mathrm{pc}$ [12], (see section 2.1]).

On 3 Mar 2005, global VLBI observations of M82 were made using a sixteen element array. This incorporated six of the EVN antennas and all of the ten VLBA antennas. The observations were made over a period of 18 hours in spectral line mode at a frequency of $1.6 \mathrm{GHz}$, using a total of 128 channels with $0.125 \mathrm{MHz}$ bandwidth each; this gives a total bandwidth of $16 \mathrm{MHz}$. Observations of the phase reference source J0958+65 were made by switching between this and M82. The sources 3C84 and J0927+39 were also observed and used in the fringe-fitting and bandpass calibration.

Despite their detection in all of the global VLBI data, the sources 44.01+59.6 and 45.17+61.2, which are also very compact, are yet to be compared over the epochs in the same way, though it is hoped this will be done in the future. Images of both of these sources from the most recent epoch can be seen in Fig. 1 . 

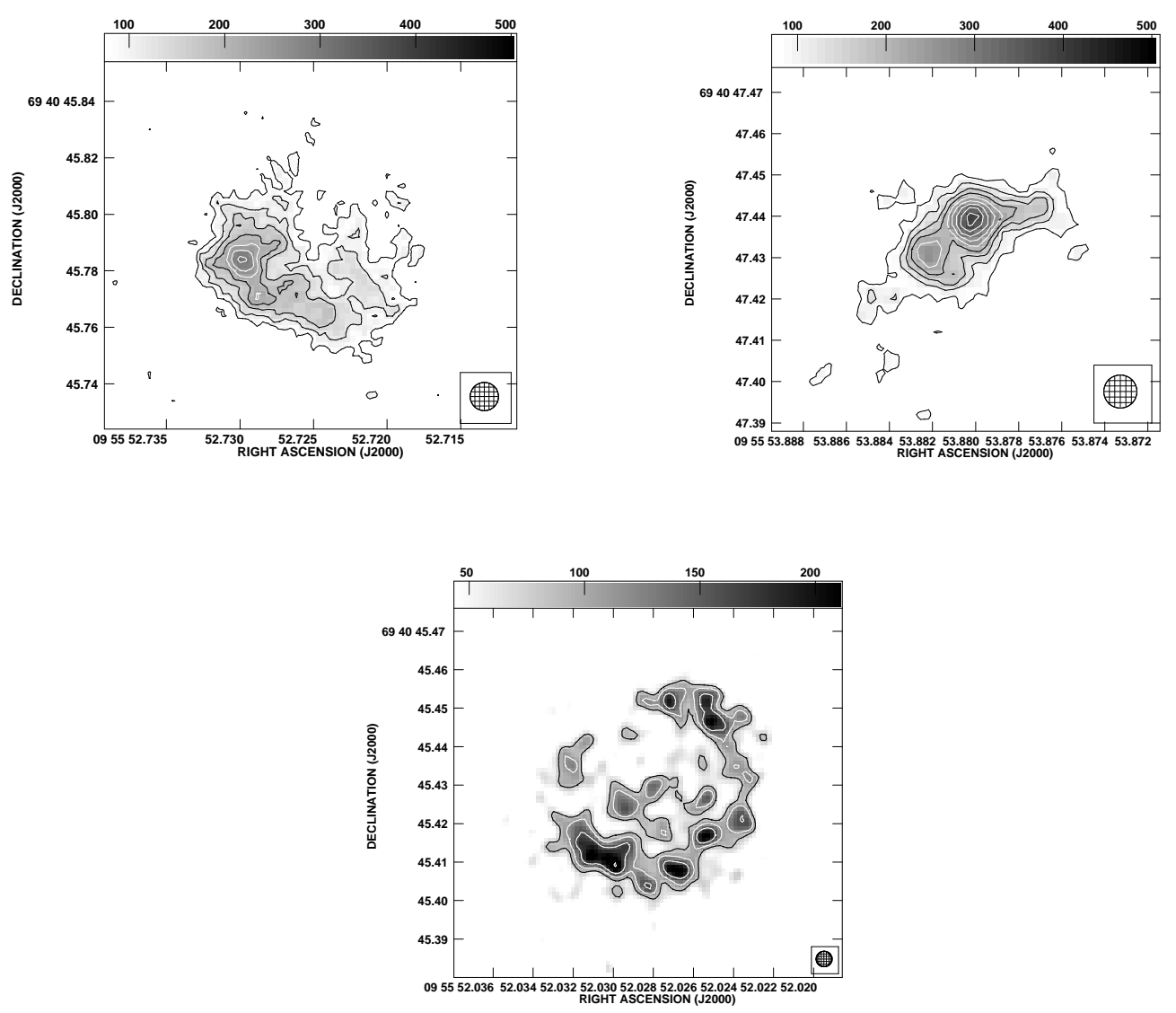

Figure 1: Grey-scale and contour images of the SNR 44.01+59.6 (left) and 45.17+61.2 (right) restored with 10 and 8 mas beams, respectively, from the 2005 global VLBI observations. Grey-scale for both images ranges linearly from 80 to $500 \mu \mathrm{Jy}_{\text {beam }}{ }^{-1}$ and the contours are plotted at $-1,1,1.5,2, . .8 .5,9 \times$ $75 \mu \mathrm{Jy}_{\text {beam }^{-1}}$. The contour and grey-scale image of $43.31+58.3$ (bottom), also from the 2005 observations, restored with a 4 mas beam is included for comparison. The contours are plotted at $-1,1,1.414,2,2.828,4,5.657 \times 80 \mu \mathrm{Jy}_{\text {beam }}{ }^{-1}$ and the grey-scale ranges from 45 to $210 \mu \mathrm{Jy} \mathrm{beam}^{-1}$.

\section{$2.141 .95+57.5$}

41.95+57.5 is the most compact source in M82 and is unusual in more than one sense. As can be seen in Fig. 2, this source has a distinct bi-polar structure, not typical of the more ring-like nature expected of supernova remnants. Observations of this source at $2.3 \mathrm{GHz}$ and $5 \mathrm{GHz}$ showed a structure indicative of an elongated shell, hence its initial classification as a SNR [13, 14]. Further observations at $1.6 \mathrm{GHz}$ using the EVN confirmed this elongated structure, sized $20 \times 10$ mas [ 10$]$. The two bright knots of radio emission were distinguishable via cross-cuts along the major axis in the 1997 EVN observations, though are not completely resolved. The subsequent global VLBI observations along with earlier observations by Wilkinson \& de Bruyn 1990 [14], Trotman 1996 [15] showed these as two distinct radio emitting regions.

The previous global VLBI observations of this source have been used to show that it has a relatively low expansion velocity of $1500 \pm 400 \mathrm{~km} \mathrm{~s}^{-1}$ [12]. This is consistent with other mea- 


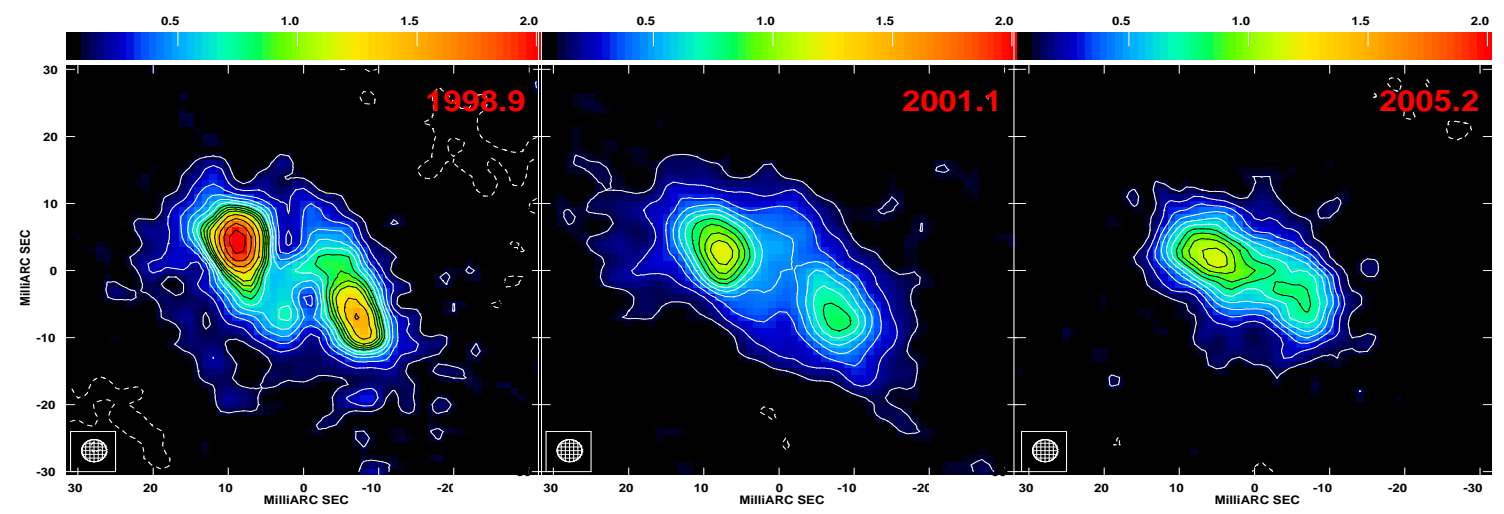

Figure 2: Contour and colour-scale images of $41.95+57.5$ restored with a 3.3 mas circular beam. Contours are plotted at $-1,1,2,3,4,5,6,7,8,9,10 \times 0.21 \mathrm{mJy}^{b^{2}} \mathrm{~m}^{-1}$ for the 1998 epoch and $\times 0.11 \mathrm{mJy}^{\mathrm{beam}}{ }^{-1}$ for epochs from 2001 and 2005 . The colour-scale is linear ranging from 0 to $2.0 \mathrm{mJy}^{\mathrm{beam}}{ }^{-1}$.

surements of expansion using EVN and global data [10, 11] giving a general expansion rate of $\sim 1500-2000 \mathrm{~km} \mathrm{~s}^{-1}$ and assuming constant expansion yields an upper limit of around 100 years for the age of $41.95+57.5$.

This source is the only one of the population observed to show any continued flux density variation. Its integrated flux density has been decreasing by around $8.5 \%$ per year since the first observations in 1965. If the age is taken to be 100 years, this flux density decrease implies that at birth it would have had a flux density $\sim 170 \mathrm{Jy}$. Muxlow et al. 2005 [16] have shown that $41.95+57.5$ makes a significant contribution to the observed flux of M82, and go on to suggest that it could be an evolved GRB, explaining its bi-polar structure and flux evolution.

The continued observations of this source at $1.6 \mathrm{GHz}$ using global VLBI have shown the structure on scales of a few mas to be evolving significantly. Its unusual structure and continued flux variability mark this source as atypical of the observed SNR in M82 to date. Of the $\sim 50$ known, no other source shows any similar characteristics to $41.95+57.5$ and further observation is required.

\section{$2.243 .31+59.2$}

$43.31+59.2$ is the most well studied of the SNR in M82. The continued observation of its expansion over a 19 year time-line provides important information on the nature of such SNR and the environments they occupy. Previous work on VLBI observations of this source such as [10, 11, 12] have shown it to be expanding at $\sim 10^{4} \mathrm{~km} \mathrm{~s}^{-1}$. The expansion can be seen most prominently in the comparison between the first two epochs of EVN observations, though the subsequent evolution has yielded consistent expansion velocities.

Thanks to the continued monitoring of this source, it is possible to fit a range of deceleration parameters to the measured sizes at each epoch. McDonald et al. 2001 [11] and Huang et al. 1994 [17] parameterise the evolution of such an object using the formula $D=k T^{\delta}$, where $\mathrm{D}$ is the size of the remnant and $\mathrm{T}$ is its age. $\delta$ represents the deceleration parameter and k is a constant. Fig. 4 shows the size measurements for each epoch with fitted deceleration curves. The actual birth date is unknown, though an upper limit can be placed on this source, as it was first observed in 1972 by Kronberg and Wilkinson [3]. This can be used to constrain the deceleration parameter. The 


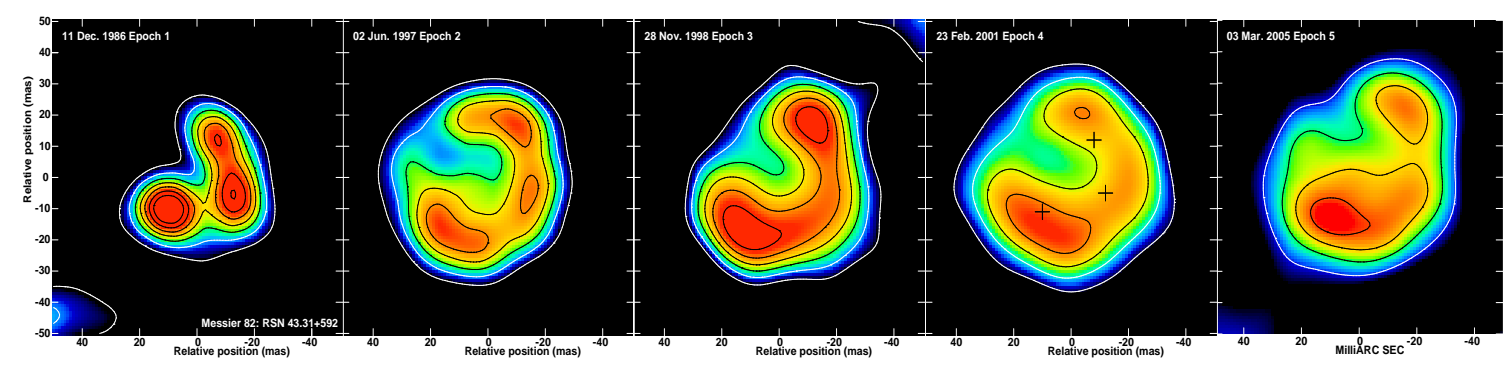

Figure 3: Contour and colour-scale images of the SNR 43.31+59.2 from each epoch of the VLBI observations. Contours are plotted at $-1,1,2,3,4,5,6,7,8,9,10 \times 0.35 \mathrm{mJy}^{-1}$ beam $^{-1}$ and the colour-scale is linear ranging from 0.1 to $2.2 \mathrm{mJy} \mathrm{beam}^{-1}$ for the first three (from the left) and from 0.1 to $1.7 \mathrm{mJy} \mathrm{beam}^{-1}$ for the other two. Previous epoch images taken from Beswick et al. 2006 [12].

measurements shown for previous epochs are taken from Beswick et al. 2006 [12] and all sizes have been measured using integrated annular profiles across the remnant using images convolved with a 15 mas beam to enable the inclusion of the EVN epochs. Those quoted are the sizes at $50 \%$ of the peak from each epoch.

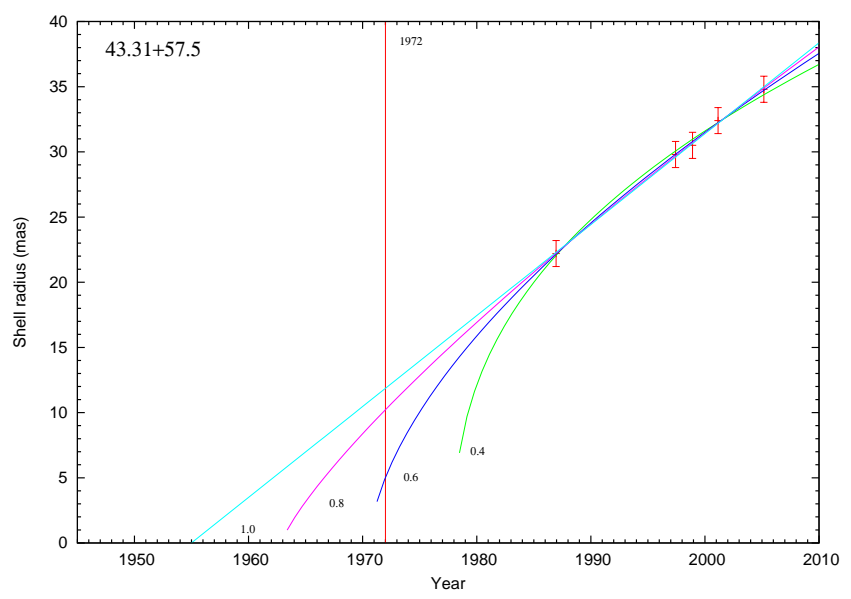

Figure 4: Measured radius of $43.31+59.2$ for the five epochs. Also plotted are four of the possible deceleration parameters fitted to the measurements. The vertical line shows the year of the first observation of 43.31+59.2. Previous epoch measurements taken from Beswick et al. 2006 [12].

$43.31+59.2$ would appear to be in, or close to, free expansion, of which its still well-defined shell structure is a strong indicator and is supported by the lack of a low frequency turnover at frequencies below $1.6 \mathrm{GHz}$ [18]. Continued observations of this source will reveal more information on the nature of its surrounding medium and will in particular enable determination of the deceleration rate.

\section{MERLIN and global VLBI combination}

Observations of M82 using the seven element MERLIN array were carried out simultaneous to the global VLBI observations in March 2005. Using the same calibration sources as for the global VLBI observations, this data has been reduced using the MERLIN pipeline and combined with the 
global VLBI dataset. This will provide images of M82 with resolutions ranging from a few mas to $\sim 100$ mas. This will enable the first detailed study of the population of discrete sources at $1.6 \mathrm{GHz}$ and such a detailed study has only been possible to date with 5-GHz observations [6, 乙, 8, 凤]
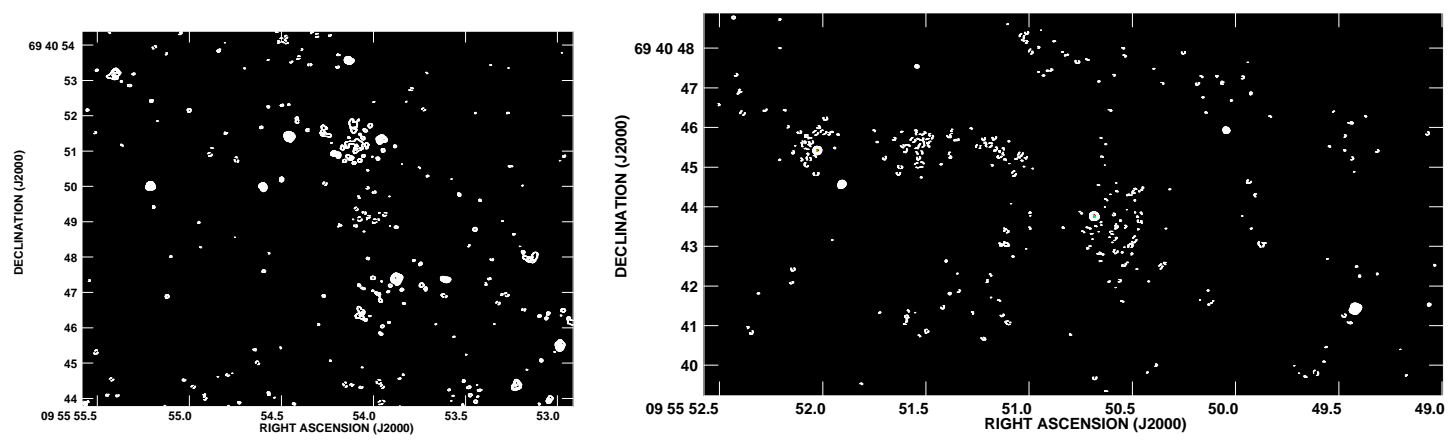

Figure 5: Example fields of the combined 1.6-GHz dataset, convolved with a 35 mas beam. Contours are plotted at $-1,1,2,3 \ldots 19,20 \times 0.196 \mathrm{mJy}_{\text {beam }}{ }^{-1}(3 \sigma \mathrm{rms})$ and the grey-scale is linear, ranging from 0.07 to $50 \mathrm{mJy}$ beam $^{-1}$.

Fig. 5 shows two example fields from images of the 1.6-GHz combined dataset convolved with a 35 mas beam. A number of compact sources are clearly visible in these fields, and in total 30 SNR and 3 HII regions (of the known sources) have been identified so far in this data.

\section{Conclusions}

The 5th epoch of global VLBI observations of M82 have been calibrated and used to produce images of four of the most compact sources in M82. In particular, the SNR 43.31+59.2 has been compared to previous VLBI observations of this source covering a 19 year timescale. Preliminary size measurements using the new dataset have provided estimates and confirmation of the expansion velocity of this source. A more in-depth study will, it is hoped, begin to constrain the nature of the expansion of this source, for example determining whether it is still undergoing free expansion. The most recent images of the source $41.95+57.5$ have shown this to be continually evolving over the timescale of the observations and further study is necessary to constrain its true nature.

For the first time, a successful combination of simultaneous MERLIN and global VLBI observations at $1.6 \mathrm{GHz}$ has provided the opportunity to study the detail of the population of compact sources in M82 at a frequency other than $5 \mathrm{GHz}$.

\section{Acknowledgments}

MERLIN is a national facility run by the University of Manchester on behalf of PPARC. The European VLBI Network is a joint facility of European, Chinese, South African and other radio astronomy institutes. The VLBA is operated by the National Radio Astronomy Observatory, a facility of the National Science Foundation, operated under cooperative agreement by Associated Universities, Inc. DF and MKA acknowledge financial support via a PPARC funded studentship. 


\section{References}

[1] E. M. Burbridge, G. R. Burbridge, V. C. Rubin, A Study of the Velocity Field in M82 and its Bearing on Explosive Phenomena in that Galaxy, ApJ 1964 (140) 942

[2] F. Bash, Brightness Distributions of Radio Sources at 2695 MHz, ApJS 1968 (149) 373

[3] P. P. Kronberg, P. N. Wilkinson, High-resolution, multi-frequency radio observations of M82, ApJ 1975 (300) 430

[4] P. P. Kronberg, P. Biermann, F. R. Schwab, The nucleus of M82 at radio and X-ray bands - Discovery of a new radio population of supernova candidates, ApJ 1985 (291) 693

[5] S.W. Unger, A. Pedlar, D.J. Axon, P.N.Wilkinson, P.N. Appleton, Young supernovae in the starbust galaxy M82, MNRAS 1984 (211) 783

[6] T.W.B. Muxlow, A. Pedlar, P.N. Wilkinson, D.J. Axon, E.M. Sanders, A.G. de Bruyn, The Structure of Young Supernova Remnants in M82, MNRAS 1994 (266) 455

[7] D. Fenech, T.W.B. Muxlow, A. Pedlar, R.J. Beswick, M.K. Argo, K.A. Wills, MERLIN Deep Radio Observations of Supernova Remnants in M82, in proceedings of Stellar End Products, Granada, April 13-15, 2005, Memorie S. A. It 2005 (76) 583

[8] T.W.B. Muxlow, A. Pedlar, A. Riley, J. McDonald, R.J. Beswick, K.A. Wills, Deep radio imaging with MERLIN of the supernova remnants in M82, in proceedings of IAU Colloquim 192, Springer Proceedings in Physics 2005 (192) 227

[9] D.Fenech, A. Pedlar, T.W.B. Muxlow, R.J. Beswick, M.K. Argo, K.A. Wills, in prep.

[10] A. Pedlar, T.W.B. Muxlow, M.A. Garret, P.J. Diamond, K.A. Wills, P.N. Wilkinson, W. Alef, VLBI observations of supernova remnants in Messier 82, MNRAS 1999 (307) 761

[11] A.R. McDonald, T.W.B. Muxlow, A. Pedlar, M.A. Garret, K.A. Wills, S.T. Garrington, P.J. Diamond, P. N. Wilkinson, Global Very Long Baseline Interferometry observations of compact radio sources in M82, MNRAS 2001 (322) 100

[12] R.J. Beswick, J.D. Riley, I. Marti-Vidal, A. Pedlar, T.W.B. Muxlow, A.R. McDonald, K.A. Wills, D. Fenech, M.K. Argo, 15 years of Very Long Baseline Interferometry observations of two compact radio sources in Messier 82, MNRAS 2006 (369) 1221

[13] N. Bartel, M.I. Ratner, A.E.E. Rogers, I.I. Shapiro, R.J. Bonometti, N.L.Cohen, M.V. Gorenstein, J.M. Marcaide, R.A. Preston, VLBI observations of 23 hot spots in the starburst galaxy M82, ApJ 1987 (323) 505

[14] P.N. Wilkinson, A.G. deBruyn, Shell-like structure in $41.9+58$, a powerful supernova remnant in M82, MNRAS 1990 (242) 529

[15] W.M. Trotman, VLBI Observations of 41.9+58: An intermediate-age radio supernova in the starburst nucleus of M82, MSc Thesis, University of Manchester 1996

[16] T.W.B. Muxlow, A. Pedlar, R.J. Beswick, M.K. Argo, T.J. O’Brien, D. Fenech, W. Trotman, Is 41.95+57.5 in M82 actually an SNR?, in proceedings of Stellar End Products, Granada, April 13-15, 2005, Memorie S. A. It 2005 (76) 586

[17] Z. P. Huang, T. X. Thuan, R. A. Chevalier, J.J. Condon, Q. F. Yin, Compact radio sources in the starburst galaxy M82 and the Sigma-D relation for supernova remnants, ApJ 1964 (140) 942

[18] K.A. Wills, A. Pedlar, T.W.B. Muxlow, P.N. Wilkinson, Low-frequency observations of supernova remnants in M82, MNRAS 1997 (291) 517 